Cahiers de recherches médiévales

Le poison et ses usages au Moyen Âge

\title{
Feminea fraus
}

Adélaïde del Vasto (ca. 1075-1118), une princesse empoisonneuse sicilienne du XII ${ }^{\mathrm{e}}$ siècle

\section{Bérangère Soustre de Condat-Rabourdin}

\section{Q OpenEdition}

12 Journals

Édition électronique

URL : https://journals.openedition.org/crm/11502

DOI : $10.4000 / \mathrm{crm} .11502$

ISSN : 1955-2424

Éditeur

Honoré Champion

Édition imprimée

Date de publication : 15 juin 2009

Pagination : 39-51

ISSN : 1272-9752

Référence électronique

Bérangère Soustre de Condat-Rabourdin, «Feminea fraus », Cahiers de recherches médiévales [En ligne], 17 | 2009, mis en ligne le 15 juin 2012, consulté le 15 décembre 2022. URL : http:// journals.openedition.org/crm/11502; DOI : https://doi.org/10.4000/crm.11502

Ce document a été généré automatiquement le 15 décembre 2022.

Tous droits réservés 


\title{
Feminea fraus
}

Adélaïde del Vasto (ca. 1075-1118), une princesse empoisonneuse sicilienne du XII ${ }^{\mathrm{e}}$ siècle

\author{
Bérangère Soustre de Condat-Rabourdin
}

\section{Introduction}

1 À l'exception d'Adélaïde del Vasto (1101-1112), de Marguerite de Navarre (1166-1171), de Sibille d'Acerra (février-décembre 1194), de Constance de Hauteville (1197-1198), de Constance d'Aragon (1210-1216), de Marie d'Aragon (1377 1392) et de Blanche de Navarre (1410-1412), qui furent toutes investies du pouvoir politique pendant leur veuvage et la minorité du prince héritier, on ignore presque tout de la personnalité, du caractère et de l'existence des autres épouses royales. Comme le rappelait Ferdinand Chalandon : « ... elle (la reine) reçoit un douaire considérable, mais, en général, elle ne paraît pas jouer un rôle important dans le gouvernement. Sauf pour Adélaïde, Marguerite et Sibille que les circonstances amenèrent à exercer la régence, nous ignorons presque tout des femmes des princes normands $»^{1}$.

Des sources littéraires de l'époque, en général assez critiques envers le pouvoir politique exercé par la souveraine, se dégage une image stéréotypée et négative de la femme d'influence: accusée des pires crimes (assassinat, corruption), elle est une véritable virago à l'affût du pouvoir et prête à tout, même au meurtre des enfants nés du premier mariage de son époux, pour assurer la succession à son fils.

3 L'origine étrangère de la plupart des épouses princières siciliennes, natives d'Angleterre, de France, d'Orient et, surtout, d'Espagne a certainement pesé lourd dans le sens de la perception négative de leur altérité ${ }^{2}$ ce qui explique par exemple la fréquence de certains thèmes, tels que celui de l'empoisonnement, dans les chroniques italo-normandes ${ }^{3}$.

Les sources médiévales de Sicile sont relativement pauvres en affaires de poison

dont les femmes de pouvoir ont été les protagonistes: des sept reines qui ont exercé la régence dans la période qui s'étend de la création du royaume (1130) jusqu'à son annexion au Royaume d'Espagne dont il deviendra vice-règne (1504), une seule est directement impliquée dans un crime de poison. Image même du 
pouvoir féminin tel que le construit la vision masculine et cléricale de l'époque ${ }^{4}$, Adélaïde del Vasto est certainement l'un des personnages les plus célèbres de l'histoire médiévale sicilienne, mais aussi l'un des plus vilipendés par ses chroniqueurs.

4 Les sources littéraires sur Adélaïde, troisième épouse de Roger I ${ }^{\mathrm{er}}$, régente du comté de Calabre et de Sicile (1101-1112), ne sont pas très abondantes. On sait qu'elle était nièce $\mathrm{du}$ marquis Boniface de la dynastie des «Aleramici » dont le domaine territorial s'étendait du Piémont méridional à la Ligurie occidentale.

Des études récentes ont prouvé que l'appellation « del Vasto » par laquelle est désignée la branche sicilienne de la famille ${ }^{5}$ n'est utilisée dans les actes qu'à partir de 1170 , c'està-dire bien après qu'elle se fut éteinte en Sicile ${ }^{6}$. À la fin du $\mathrm{X}^{\mathrm{e}}$ siècle, ce lignage s'était divisé en deux branches qui furent placées sous l'autorité des deux fils du comte et marquis des «Aleramici », Odon et Anselme : de la première descendent les marquis de Montferrat (Piémont) et, de l'autre, Boniface, oncle d'Adélaïde et de son frère, Henri, et les marquis de Saluzzo.

6 Adélaïde est mentionnée pour la première fois dans le De rebus gestis Rogerii Calabriae et Siciliae comitis et Roberti Guiscardi ducis fratris eius ${ }^{7}$. Son auteur, le moine Geoffroi Malaterra, y raconte que, veuf de sa deuxième épouse la Normande Éremburge de Mortain, Roger $\mathrm{I}^{\mathrm{er}}$ avait épousé Adélaïde :

Anno igitur MLXXXIX comes Rogerius, uxore Eremburga, filia Guillelmi comitis Mortonensis, defuncta, aliam duxit Adelaydem nomine, neptem Bonifacii famosissimi Italorum marchionis, filiam videlicet fratris eius, iuvenculam honestae admodum faciei ${ }^{8}$.

7 Malaterra situe le mariage en 1089 : cette date est cependant très controversée par les historiens. Il est connu en effet que le moine fondait ses récits sur des sources orales ${ }^{9}$, ce qui expliquerait l'imprécision de ses indications chronologiques. La plupart des événements qu'il relate sont toujours antidatés d'une année par rapport aux autres sources de l'époque. Le mariage a vraisemblablement eu lieu entre 1089 et 1090 ; Adélaïde, qui était aux dires de Malaterra iuvencula et puella, devait avoir alors approximativement une douzaine d'années, âge auquel les jeunes filles étaient autorisées par le droit canonique à se marier ${ }^{10}$. En poursuivant sa narration, il explique également que le comte avait donné en mariage deux sœurs d'Adélaïde à ses deux fils, Geoffroi et Jourdain, et que le premier avait renoncé aux noces car atteint d'un mal incurable :

... duasque sorores eiusdem puellae duobus filiis suis, Gaufredo videlicet et Jordano, in matrimonium copulavit. Sed Gaufredus, antequam nubiles annos attingisset, quod dolor est dicere, morbo prohibente minime eam cognovit. Jordanus autem solemnibus nuptiis duxit ${ }^{11}$.

Quelques actes de donation, souscrits par Roger $\mathrm{I}^{\mathrm{er}}$ au cours de ses voyages, évoquent la présence d'Adélaïde aux côtés de son époux lors de ses déplacements en Calabre et en Sicile $^{12}$.

9 De son union avec Roger ${ }^{\mathrm{e} r}$, Adélaïde eut deux fils : Simon, né entre 1093 et 1094, et Roger II, dont elle accoucha en 1095. Elle aurait également eu deux filles, mais Geoffroi Malaterra ne nous livre aucun renseignement sur elles, se contentant de ne mentionner que les filles de Roger ${ }^{\text {er }}$ qui avaient contracté des unions matrimoniales prestigieuses $^{13}$.

10 À la mort de Roger $\mathrm{I}^{\mathrm{er}}$ survenue à Mileto (Calabre) en juin $1101^{14}$, Adélaïde devint régente du comté de Calabre et de Sicile au nom de son fils Simon qui avait été désigné pour succéder à son père ${ }^{15}$. 
11 D'après Carlo Alberto Garufi, c'est Adélaïde elle-même qui aurait tout fait pour assurer la succession de ses fils, Simon et Roger, au détriment des enfants nés des précédents mariages du comte, et notamment de Geoffroi qui était atteint de lèpre ${ }^{16}$. C'est dire comment cette femme de pouvoir a souffert de jugements négatifs, et cela même à une époque relativement récente ; les formules à l'emporte pièce et les images stéréotypées dont use Garufi sont symboliques de la perception négative du pouvoir féminin dans les sources médiévales, mais aussi dans l'historiographie du XIXe et du XXe siècle.

Simon décède prématurément le 28 septembre $1105^{17}$. La succession paternelle est alors ouverte à son frère Roger, le futur Roger II, premier roi de Sicile, qui était encore mineur. C'est donc sa mère qui continue à exercer la régence au nom de son fils.

Nous savons peu de choses sur les onze années de la régence d'Adélaïde, ce qui s'explique principalement par l'absence d'historiens de l'Italie méridionale pour les premières décennies du XII ${ }^{e}$ siècle. L'œuvre de Geoffroi Malaterra s'achève en 1098.

L'Ystoria Rogerii Regis Sicilie Calabrie atque Apulie, que Mathilde, l'une des sœurs de Roger, commanda à Alexandre, abbé de Saint-Sauveur de Telese en 1130, ne commence qu'à partir des années 1127. En effet si dans le préambule de l'œuvre, l'auteur déclarait qu'il allait raconter l'histoire du roi de Sicile depuis son enfance, en réalité il ne décrit que brièvement l'adolescence de Roger II, en survolant la régence d'Adélaïde qu'il évoque sous les traits d'une mulier prudentissima. Elle est également passée sous silence dans la chronique de l'Anonyme Vatican, qui fut vraisemblablement rédigée à la fin du règne de Roger ou au début du suivant ${ }^{18}$

\section{L'empoisonnement de Robert de Bourgogne : crime de pouvoir ou crime de genre?}

Le seul témoignage sur les onze années de gouvernement d'Adélaïde est celui d'Orderic Vital.

Dans son Historia ecclesiastica, le moine normand raconte comment, s'étant aperçue qu'elle ne parvenait pas à exercer seule la régence de l'immense territoire que lui avait laissé son époux, la comtesse avait fait appel à Robert, fils du duc de Bourgogne en lui donnant l'une de ses filles en mariage :

Postquam Rogerius senex, Siciliae comes, Tancredi de Altavilla filius, obiit, uxor eius Adeles cum parvulo filio regere se non posse magnas possessiones perspexit et anxia, quid agendum foret, tam secum quam cum familiaribus suis sollerter indagavit. Ingentes enim provincias prefatus consul et XI fratres eius bellica virtute optinuerant et barbaros sub potenti manu excelsi Dei fortiter in Apulia et Sicilia subegerant. Tandem prefata mulier Rodbertum, Rodberti ducis Burgundiae filium, in amicitiam copulavit eique filiam suam coniugem cum toto Sicanie principatu tradidit. ${ }^{19}$

17 Pendant que la comtesse s'occupait de l'éducation de son fils, c'est son gendre qui aurait assumé la défense militaire du vaste comté en y guerroyant avec vaillance ${ }^{20}$. En poursuivant sa narration, Orderic Vital explique qu'à la majorité de Roger, Adélaïde s'était libérée de Robert au moyen d'un breuvage empoisonné :

... et ius patris regendum tironem idoneum agnovit, egregium Francigenum probumque militem, generum suum, venenosa potione proh dolor infecit. Extincto itaque femina fraude nobili marchisio, Rogerius successit ${ }^{21}$.

18 Quelle est la part de vérité dans cette histoire? Pour la déterminer, il convient tout d'abord de distinguer ce qui relève de la réalité de ce qui n'est qu'une pure invention de 
l'historien normand. Le thème de l'empoisonnement est assez récurrent dans l'Historia ecclesiastica: plusieurs princesses y sont accusées d'avoir perpétré le crime de poison, généralement, dans le cadre familial.

Or on est frappé par la ressemblance d'Adélaïde avec une autre femme de pouvoir très influente dont le moine normand a laissé un portrait assez sombre: Sichelgaite, deuxième épouse de Robert Guiscard. D'après Orderic Vital, la princesse de Salerne aurait tenté d'assassiner Bohémond, né d'un premier mariage, afin d'assurer la succession au duché de Pouille à son propre fils, Roger Borsa.

Experte dans la préparation de poisons dont elle apprit l'art au contact des médecins salernitains, Sichelgaite prépara une potion empoisonnée qu'elle fit boire au jeune prince qui sombra dans le coma. Le crime ayant été découvert par son époux qui la menaça alors de la passer au fil de l'épée en cas où son fils décèderait ${ }^{22}$, elle prépara un antidote. Elle parvint alors à sauver Bohémond avec l'aide des médecins de Salerne, mais, rongée par le sentiment de vengeance, elle finit par empoisonner son époux quelques années plus tard.

21 Robert Guiscard ne mourut pas d'empoisonnement et il est fort probable que la tentative d'assassinat à l'encontre de Bohémond soit une invention de la part du chroniqueur.

Malgré les éléments fictifs qui caractérisent son style dans lequel le vrai est «mescolato al fantastico, l'erroneo all'esatto " ${ }^{23}$, le récit d'Orderic contient des éléments véridiques, en particulier le caractère rigoureux d'Adélaïde et sa fermeté dans le domaine politique.

Robert de Bourgogne, victime de la feminea fraus commise par Adélaïde, ne nous est connu qu'à travers Orderic Vital. Aucune autre source n'attestant de son existence, l'historien Michele Amari (1806-1889) considérait que ce personnage était une pure invention de la part du chroniqueur normand qui était « frate ghiotto di favole ed avverso all'Italia $»^{24}$.

La connaissance des faits italo-méridionaux de la part d'Orderic Vital ne fait cependant aucun doute.

Le moine rédigea son Historia ecclesiastica depuis l'abbaye de Saint-Évroult en fondant son récit principalement sur les informations des moines qui revenaient d'Italie méridionale: Ernaldus de Le-Tilleul25, qui s'était rendu en Pouille, en Calabre et en Sicile où résidaient certains de ses parents pour leur demander des donations en faveur de son monastère ; Guillaume, son frère, devenu abbé du monastère calabrais SainteEufémie qui avait été fondé par Robert Guiscard ${ }^{26}$, et l'un de leurs cousins, Rainaldus d'Échaufour, également moine à Saint-Évroult, qui, au cours d'une mission qui lui avait été confiée par l'abbé Roger (1091-1103) avait séjourné presque trois ans au monastère Sainte-Eufémie :

... atque Apuliam et Calabriam Siciliamque, ut de spoliis parentum suorum ecclesiae suae subsidium suppeditaret penetravit. Tunc Guillemum, abbatem sanctae Eufemiae, fratrem suum, et Guillelmum de Grentemaisnil consobrinum suum aliosque cognatos suos in Italia locupletatos adiit ${ }^{27}$.

De celui-ci, Orderic loue la mémoire prodigieuse des choses vues et entendues : Memoriae vero narrandi quae viderat vel audierat magnifice viguit, delectabilique relatu rerum, quas in divinis voluminibus seu peritorum assertionibus rimatus est, socios multoties lenivit ${ }^{28}$.

L'un des plus fidèles collaborateurs de la comtesse, dans les années de sa régence, était Robert Borrel ${ }^{29}$, cadet du duc Robert de Bourgogne (1032-1076) qui avait été contraint à 
s'exiler en raison de divergences avec son neveu Hugues, duc de Bourgogne ${ }^{30}$. Orderic Vital aurait donc fait un amalgame entre celui-ci et Robert Borrel.

\section{La régence : un gouvernement de consolidation et d'équilibre}

La politique d'Adélaïde reprit dans les grandes lignes celle de son époux : elle sut mettre à profit ce qu'elle avait appris à ses côtés en mettant en place un gouvernement de " consolidation et d'équilibre », même si elle sut faire preuve d'une certaine dureté.

Un document, daté de 1123, décrit comment la veuve avait ordonné l'assassinat d'un groupe de barons rebelles qui avaient occupé et détruit le château de Focerò, près de Patti (province de Messine) ${ }^{31}$.

Ordéric Vital raconte que la "veuve impitoyable » broyait ses ennemis en morceaux pour réprimer les révoltes qui éclataient en Calabre et en Sicile. Dans les années troublées qui suivirent la mort de Roger I ${ }^{\text {er }}$, l'une des préoccupations majeures de la comtesse fut de s'entourer de collaborateurs fidèles et dévoués.

$\mathrm{Au}$ cours de sa régence, son frère, le comte Henri fut investi de la puissante seigneurie qui était formée des comtés de Paternò et de Butera, tandis que la puissante famille du comte Robert Avenel, qui plus tard sera apparentée à Roger II, reçut la seigneurie d'un vaste ensemble de terres situées dans le Val Demone et dont le centre se trouvait à Collesano.

31 Ces territoires formaient ce que les historiens italiens ont qualifiés de «colonies lombardes » (Enna, Nicosia, Montalbano, Novara et Randazzo), des bourgs situés dans une zone à prédominance italo-grecque qui devait être progressivement latinisée par les nouvelles populations ${ }^{32}$.

2 Dans l'entourage de la comtesse, se distinguent également les Normands Josbert de Lucy et Guillaume de Hauteville, neveu de Roger I ${ }^{\text {er }}$, qui, comme Robert Avenel, s'étaient déjà illustrés au service de son époux ${ }^{33}$. À ceux-ci étaient associés les ItaloGrecs, Nicolas préposé au trésor comtal entre 1090 et 1105 ; le logothète (chef de cabinet) Léon, et l'amiral Christodoule qui semble avoir exercé une grande influence sur le jeune Roger $\mathrm{II}^{34}$; celui-ci connaissait davantage le grec que le latin, ce qui se justifierait par le fait que le prince avait grandi dans la zone orientale de l'île, qui était de langue et de culture grecques.

33 Adélaïde fit transférer le siège du comté de Troina à Messine ${ }^{35}$ : c'est là que fut établi la plupart de ses actes en faveur des monastères grecs du Val Demone dont elle était l'une des bienfaitrices les plus importantes ${ }^{36}$.

Entre mars et juin 1112 à l'époque de la majorité de Roger II (... filius meus, Dei gratia iam miles, iam comes Sicilie et (alabrie), Adélaïde déplaça la cour comtale de Messine à Palerme $^{37}$, la plus grande ville de l'île, dont la population se divisait en deux ethnies ${ }^{38}$ : l'une grecque et, l'autre, majoritaire, de langue arabe et de religion musulmane. Le choix de cette ville fut capital pour l'histoire normande: en effet, jusque là, les Normands ne s'étaient établis que dans des châteaux et des petits centres fortifiés.

La décision de la régente posa les fondations pour l'établissement d'une monarchie qui aurait été caractérisée, plus tard, par une structure centralisée avec à sa tête un groupe dirigeant formé de grecs et d'arabes. 
36 À la fin de sa régence, Adélaïde reçut, au printemps de l'année 1110, Sigurd de Norvège qui se rendait alors en Terre sainte : le séjour sicilien du roi norvégien est raconté par le chroniqueur islandais Snorri Sturluson (1179-1241) qui décrit, dans son œuvre intitulée Heimskringla, une histoire universelle sur les dynasties royales norvégiennes, comment, pendant ce voyage, Sigurd aurait intronisé symboliquement Roger II ${ }^{39}$.

\section{Un mariage dicté par la raison d'État}

Les dernières années de régence d'Adélaïde sont marquées par son mariage (1113-1117) avec Baudouin $\mathrm{I}^{\mathrm{er}}$, roi de Jérusalem (1058-1118). Les deux historiens des croisades Albert d'Aix-la-Chapelle et Guillaume de Tyr racontent qu'au cours de la deuxième moitié de 1112, le souverain avait dépêché une mission diplomatique à Palerme pour demander la main d'Adélaïde. Tout en sachant que le roi était déjà marié, elle accepta de l'épouser, mais à une condition : si aucun enfant ne venait à naître, la couronne reviendrait à son fils Roger à la mort du roi :

Forma autem conditionum hec erat, quod si rex ex predicta comitissa prolem susciperet, ei post regis obitum sine contradictione et molestia regnum concederetur, quod si absque herede ex eadem comitissa suscepto defungeretur, comes Rogerius, filius eius, heres existeret et in regno sine contradictione et molestia rex futurus succedere $t^{40}$.

Il s'agissait pour les deux époux d'un mariage de convenance : Baudouin se procurait la riche dot de la douairière de Sicile pour régler la solde de son armée et Adélaïde offrait la couronne de Jérusalem à son fils.

Ordéric Vital raconte que la veuve rusée et assoifée d'argent et de pouvoir avait amassé une grande quantité de biens à la mort de son mari s'adonnant à une vie de luxure en Terre Sainte :

Callida mater eius, quae filia (!) Bonifacii Liguris fuerat, a morte mariti sui pecuniis undecumque collectis ingentem thesaurum sibi congesserat. Quod audiens Balduinus prior Ierosolimae rex opes concupivit ipsamque, ut coniugali ritu sibi copularetur, per illustres procos requisivit. Illa vero fastus et honoris insatiabiliter avida nobilibus paranimphis adquievit et, multitudine stipata clientum, cum ingenti aerario Ierusalem properavit ${ }^{41}$.

Les noces furent célébrées en grande pompe à Jérusalem en septembre 1113, mais leur union resta stérile :

Missi igitur legati conditiones predicta gratanter suscipiunt et interpositis iuramentis, prout exigebantur, quod his pactis a domino rege et suis principibus bona fede, sine fraude et dolo malo staretur, comitissa, filio universa necessaria suggerente, se accingit ad iter, et oneratis navibus frumento, vino et oleo et salsis carnibus, armis pretera et equitaturis egregiis, assumens secum infinitam pecuniam, omnibus eram prosequentibus copiis, in nostram, ut predictam est, apulit regionem ${ }^{42}$.

À la suite de l'intervention du patriarche de Jérusalem qui avait invoqué la parenté du roi avec la comtesse de Calabre et et de Sicile, Adélaïde fut répudiée. Elle quitta Jérusalem le 25 avril 1117, dolens igitur et trististam de illata contumelia quam de opibus inutiliter consumptis ${ }^{43}$.

Rentrée en Sicile, elle se retira au couvent du Saint-Sauveur de Patti (province de Patti) où elle décèda un an après (16 avril 1118 $)^{44}$.

Même si Roger n'était pas parvenu à hériter du royaume de Terre Sainte, Adélaïde contribua indéniablement à accroître la puissance du comté de Calabre et de Sicile, en posant les fondements politiques de celui qui serait devenu l'un des plus puissants royaumes de l'Occident médiéval. 


\section{Une terre de poisons et d'empoisonneuses} médiévale, la Sicile, comme l'Italie méridionale, était réputée pour abriter des experts en fabrication de poisons et dans l'eruditio veneficiorum, science qu'ils auraient apprise au contact des musulmans qui habitaient l'île. Une célèbre enluminure du Liber ad Honorem Augusti, écrit à Palerme à la fin du XII ${ }^{e}$ siècle par le poète campanien Pierre de Eboli ${ }^{46}$, illustre l'importance de la science arabe à la cour sicilienne: dans l'image qui figure le deuil du populus trilinguis pour la mort de Guillaume II, c'est un médecin musulman (Achim medicus) qui veille au chevet du roi moribond, tandis qu'un astrologue (astrologus), également musulman, observe les astres ${ }^{47}$. Le rôle des médecins arabes dans la médecine médiévale de l'Occident est connu ${ }^{48}$ : en Italie méridionale et en Sicile, ils eurent un rôle important dans l'établissement des premières écoles de médecine (École médicale de Salerne ${ }^{49}$, Université Frédéric II à Naples), mais ils furent également très présents à la cour de Frédéric II et de ses descendants ${ }^{50}$. À côté de ce savoir médical érudit se développa un savoir populaire dans lequel les frontières entre science et magie, savoir et ignorance n'étaient pas toujours clairement définies, et où le poison joua une part importante.

Trois affaires de crime de poison, qui eurent lieu entre le $\mathrm{XVI}^{\mathrm{e}}$ et le $\mathrm{XVIII}^{\mathrm{e}}$ siècle, illustrent parfaitement la réputation de terre "vénénifère » dont la Sicile souffrait depuis le Moyen Âge. Thofania d'Adamo ( $† 1633)$, Giulia Tofano ( $\uparrow 1659)$ et Giovanna Bonnano $(\dagger 1789)$, toutes les trois originaires de Palerme, sont l'image même du « topos » littéraire de l'empoisonneuse/sorcière telle que le livre la tradition populaire de tous temps: des femmes mauvaises et animées par l'instinct de détruire avec la « malefica arte ${ }^{51}$. mari et de plusieurs personnes cum veneno propinato.

(1632-1639), plusieurs autres personnes furent arrêtées et condamnées à la peine capitale pour des affaires criminelles liées au poison ${ }^{52}$ : Francesca la Sarda ou Rapisardi et Placido di Marco qui furent exécutés, l'une, par décapitation et, l'autre, par écartèlement, pour avoir fabriqué des poisons hautement toxiques dont l'ingestion provoquait le décès en quelques jours.

47 Ce poison n'était pas l'invention des deux accusés : avant de mourir, interrogé sous la torture, Placido di Marco accusa Thofania d'Adamo de l'invention de la terrible potion dont elle transmit la recette à sa fille, Giulia Tofano. Celle-ci s'établit à Rome où elle devint très riche avec le commerce de ce poison appelé « Acqua Toffana », petite eau de Naples ou manne de Saint Nicolas. Destiné essentiellement aux femmes qui souhaitaient mettre fin à des unions malheureuses - la revendication «sociale » de l'empoisonnement de la part des accusées est commune aux trois affaires, le poison était vendu en petites bouteilles à l'effigie de saint Nicolas. Il était composé d'arsenic, de plomb et d'antimoine mélangés avec de l'eau; c'est le cardinal Pietro Sforza Pallavicino (1607-1667) qui nous en a transmis la recette et la préparation :

Due once di arsenico, un grosso di piombo e una sfoglietta d'acqua. Si acciacca il piombo o l'antinomio e unito all'arsenico si mette a bollire in una pignatta nova otturata bene che non sfiati, sino a che cali un dito, e che si tiene serrata con pasta o 
con sapone, e, dopo che è stata al fuoco a bollire, la composizione dell'acqua è venuta chiara e pulita ${ }^{53}$. couches les plus démunies de la société sicilienne des XVI ${ }^{e}-\mathrm{XVIII}{ }^{\mathrm{e}}$ siècles: entre le Moyen Âge et l'Époque Moderne, le profil sociologique de l'empoisonneuse change donc radicalement: dans un premier temps crime distinctif des élites féminines qui le choisissaient pour favoriser l'ascension de leurs fils au détriment de la filiation du premier lit de leur époux, l'empoisonnement fut par la suite l'affaire de marginales de toute sorte (voleuses à la petite semaine, prostituées et mendiantes).

51 Ce qui ne changea pas cependant fut le sexe des accusés: dans l'ensemble des cas attestés pour les deux périodes, seules deux affaires mettent en scène des hommes, la majorité des homicides par empoisonnement ayant été pratiquée par des femmes. Si la Sicile peut être considérée comme une terre vénénifère, il n'en demeure pas moins que l'empoisonnement fut ici, plus que partout ailleurs, un crime féminin par excellence.

\section{NOTES}

1. F. Chalandon, Histoire de la domination normande en Italie et en Sicile, Paris, A. Picard, 1907, vol. 2, p. 625.

2. F. Hartog, Le miroir d'Hérodote : essai sur la représentation de l'autre, Paris, Gallimard, 1981.

3. J. C. Russell, "Allegations of Poisoning in the Norman World», Twelfth Century Studies, New York, AMS Press, 1973, p. 83-93 ; D. Lett, «Les épouses de l'aristocratie anglo-normande des XI ${ }^{\mathrm{e}}$ $\mathrm{XII}^{\mathrm{e}}$ siècles d'après l'Histoire ecclésiastique d'Orderic Vital ", Le mariage au Moyen Âge (XI ${ }^{e}-\mathrm{XV}^{e}$ siècle), Clermont-Ferand, Université de Clermont-Ferrand, 1997, p. 15-27, F. Collard, « Une arme venue d'ailleurs. Portrait de l'étranger en empoisonneur", dans L'étranger au Moyen Âge. Actes du XXX ${ }^{e}$ Congrès de la Société des historiens médiévistes de l'enseignement supérieur public (Göttingen, 30 juin 1999), Paris, Publications de la Sorbonne, 2000, p. 95-106 ; Id. » Venenosa mulier coronata. Variations sur la figure empoisonneuse de la reine dans l'Occident médiéval », dans Reines et princesses au Moyen Âge. Actes du cinquième colloque international (Montpellier, 24-27 novembre 1999), Montpellier, Publications de l'Université Paul-Valéry-Montpellier III, 2001, vol. 1, p. 303-322.

4. M.-Th. D’Alverny, «Comment les théologiens et les philosophes voient la femme ", Cahiers de civilisation médiévale, 20, 1977, p.105-129; H. R. Bloch, Medieval Misogyny and the Invention of Western Romantic Love, Chicago, University of Chicago Press, 1991.

5. C. A. Garufi, « Gli Aleramici e i Normanni in Sicilia e nelle Puglie. Documenti e ricerche », dans Studi per il Centenario della nascita di Michele Amari. Scritti di filologia e storia araba, Palerme, 
Stabilimento tipografico Virzì, 1910, vol. 1, p. 43-87 ; G. Villari, « La corte aleramica ovvero come s'assise l'Aleramico sul trono di Paternò », dans Id., Primati etnei, Syracuse, Ediprint, 1991, H. Bresc, « Gli Aleramici in Sicilia : alcune nuove prospettive », dans R. Bordone (éd.), Bianca Lancia d'Agliano fra il Piemonte e il Regno di Sicilia. Atti del Convegno (Asti-Agliano, $28-29$ avril 1990), Alessandria, Dell'Orso, 1992, p. 147-163; F. Barone, Una famiglia comitale lombarda nella Sicilia normanna : storia e potere degli Aleramici, signori di Butera, Paternò e Cerami (1095-1161), mémoire de maîtrise, sous la direction de P. Corrao (Université de Palerme 20 avril 2000).

6. R. Bordone, «Affermazione personale e svilupi dinastici del grupo parentale aleramico: il marchese Bonifacio del Vasto (sec. XI-XII)», dans Formazione e strutture dei ceti dominanti del medioevo : marchesi, conti e visconti nel regno italico (sec. IX-XII). Atti del primo convegno (Pisa, 10-11 mai 1983), Rome, Istituto storico per il Medio Evo, 1988, p. 29-44 ; L. Provero, Dai marchesi del Vasto ai primi marchesi di Saluzzo. Sviluppi signorili entro quadri pubblici (secoli XI-XII), Turin, Deputazione subalpina di Storia Patria, 1992 (Biblioteca storica subalpina, CCIX); G. Sergi, "Dinastie e territorio : tre modelli ", dans Id., I confini del potere. Marche e signorie fra due regni medievali, Turin, Einaudi, 1995, p. 39-5; R. Merlone, Gli Aleramici. Una dinastia dalle strutture pubbliche ai nuovi orientamenti territoriali (secoli IX-XI), Turin, Deputazione subalpina di Storia Patria, 1995.

7. Sur Adélaïde, C. A. Garufi, « Adelaide nipote di Bonifazio del Vasto e Goffredo figliuolo del gran conte Ruggiero », dans Rendiconti e memorie della Reale Accademia di Scienze, Lettere ed Arti dei Zelanti di Acireale, classe di lettere, terza serie, 4, 1905, p. 185-216 ; E. Pontieri, « La madre di re Ruggero ; Adelaide del Vasto, contessa di Sicilia, regina di Gerusalemme (?-1118)», dans Atti del Convegno internazionale di studi ruggeriani (Palerme, 21-25 avril 1954). VII Centenario della morte di Ruggero II, Palerme, Società siciliana per la Storia patria, Tipografia Boccone del povero, 1955, vol.2, p. 327-432 ; H. Houben, « Adelaide del Vasto nella storia del regno normanno di Sicilia », dans Id., Mezzogiorno normanno-svevo. Monasteri e castelli, Ebrei e Musulmani, Naples, Liguori, 1996 (Nuovo Medioevo), p. 81-113.

8. Geoffroi Malaterra, De rebus..., éd. cit., IV, 14, p. 93.

9. E. Pontieri, « Prefazione », dans ibid., p. XIV-XXVIII ; H. Houben, « Adelaide... », p. 85.

10. R. Metz, «L'enfant dans le droit canonique médiéval. Orientations de recherches ", L'enfant. Vol. 2. Europe médiévale et moderne, Bruxelles, Société Jean Bodin pour l'histoire comparative des institutions, 1976, p. 9-96, p. 31-35.

11. Geoffroi Malaterra, De rebus..., éd. cit., IV, 14, p. 93.

12. E. Pontieri, «La madre... », p. 355 , note 2.

13. Adélaïde aurait été mère de Maximilla, épouse du roi Conrad $\mathrm{I}^{\mathrm{er}}$. Sur ce point, W. Holtzmann, « Maximilla regina, soror Rogerii regis », Deutsches Archiv für Erforschung des Mittelalters,19, 1967, p. 149-167 ; H. Houben, « Adelaide... », p. 87, p. 100 note 96.

14. Il Libro del Capitolo del monastero della SS. Trinità di Venosa (Cod. Casin. 334) : una testimonianza del Mezzogiorno normanno, éd. H. Houben, Galatina, Università degli Studi di Lecce, Dipartimento di Scienze storiche e sociali, 1984, p. 141.

15. Dans une charte du 22 juillet 1095 signée en faveur de l'église Sainte-Marie de Roccella (province de Catanzaro), Simon est mentionné comme filius Rogerii comitis et heres: Regii neapolitani archivi monumenta, Naples, Regia typographia, 1857/1861, vol. 6, annexe 8, p. 160.

16. C. A. Garufi, "Adelaide nipote...", p. 185-216, p. 214 : "assicurare ai suoi figliuoli, Simone prima, e morto questi, a Ruggero, la corona comitale di Sicilia e di Calabria ».

17. Sur la mort de Simon, Romuald de Salerne, Chronicon, éd. cit., p. 202 ; E. Winkelmann, «Reisefrüchte aus Italien und anderes zur deutsch-italischen Geschichte», Forschungen zur deutschen Geschichte, 18, 1878, p. 479-492, p. 473.

18. Anonyme Vatican, Historia sicula a Normannis ad Petrum Aragonensium, éd. L. A. Muratori, Milan, Typis Societatae palatinae, 1726 (Rerum italicarum scriptores, 8), col. 745-780; F. Chalandon, Histoire..., vol. 1, p. XXXVII, W. Holtzmann, «Italien (1050-1125) », Deutschlands Geschichtsquellen im Mittelalter, Die Zeit der Sachsen und Salier, Köln/Vienne, Böhlau, 1971, p. 841-936, p. 915. 
19. Orderic Vital, Historia ecclesiastica, éd. M. Chibnall, Oxford, Clarendon Press, 1969/1986 (The Ecclesiastical History of Orderic Vitalis), 6, XIII 15, p. 428.

20. Ibid., p. 434 : Rodbertus autem Burgundio, ut predictum est, filiam Rogerii Normanni coniugem habuit et principatum contra cunctos per annos $X$ strenue defensavit. Interea socrus eius Rogerium puenum educavit, atque ubi eundem ad arma gerenda.

21. Ibid.

22. Ibid., III, 7, p. 428 : Audis, Sichelguada! Per hoc sanctum Evangelium iuro quod si Buamundus filius meus hoc morbo, quo detinetur, mortuus fuerit, hoc ense interficiam te.

23. E. Pontieri, « La madre... », p. 454.

24. M. Amari, Storia dei Musulmani di Sicilia. Seconda edizione modificata e accresciuta dall'autore, notes de C. A. Nallino, Catane, R. Prampolini, 1933/1939, vol. 3, 2, p. 354.

25. H. Wolter, Ordericus Vitalis ein Beitrag zur kluniazensischen Geschichtsschreibung, Wiesbaden, F. Steiner, 1955, p. 101.

26. L.-R. Ménager, "Les fondations monastiques de Robert Guiscard", dans Quellen und Forschungen aus italienischen Archiven und Bibliotheken, 39, 1959, p. 1-116, p. 20.

27. Orderic Vital, Historia ecclesiastica, éd. cit., 4, VIII 3, p. 142. Sur Guillaume de Grandmesnil : L.R. Ménager, «Inventaire... », p. 316-317.

28. Orderic Vital, Historia ecclesiastica, éd. cit., 2, III, p. 126.

29. Quatre actes émis par Adélaïde mentionnent Robert Borrel parmi ses collaborateurs : I documenti inediti dell'epoca normanna in Sicilia, éd. C. A. Garufi, Palerme, Società siciliana per la Storia Patria, 1899, p. 511; K. A. Kehr, Die Urkunden der normannisch-sizilischen Könige: eine diplomatische Untersuchung, Aalen, Scientia Verlag Aalen, 1962, p. 413 ; Rogerii II. regis diplomata latina, éd. C. Brühl, Köln/Vienne, Böhlau, Verlag, 1987, p. 4-6, p. 6-8. Sur la biographie de Robert Borrel : Th.Kölzer, Urkunden und Kanzlei der Kaiserin Konstanze Königin von Sizilien, 1195-1198, Köln/ Vienne, Böhlau, Verlag, 1983, p. 122, note 27.

30. Orderic Vital, Historia ecclesiastica, éd. cit., 6, p. 430 notes 2 et 3. Voir aussi, J. Richard, Les ducs de Bourgogne et la formation du duché $d u \mathrm{XI}^{e}$ au XIV siècle, Dijon, Imprimerie Bernigaud \& Privat, 1954, p. 14, p. 152.

31. I diplomi greci ed arabi di Sicilia, pubblicati nel testo originale, tradotti ed illustrati da Salvatore Cusa, éd. S. Cusa, Palerme, Tipografia di Lao, 1868, p. 334 ; p. 471.

32. I. Peri, «La questione delle colonie lombarde in Sicilia ", dans Bollettino storico-bibliografico subalpino, 57, 1959, p. 253-280 ; A. Varvaro, Lingua e storia in Sicilia. I. Dalle guerre puniche alla conquista normanna, Palerme, Sellerio, 1981 ; S. Tramontana, «Popolazione, distribuzione della terra e classi sociali nella Sicilia di Ruggero il Granconte ", dans Ruggero il Gran Conte e l'inizio dello stato normanno. Atti delle seconde giornate normanno-sveve, (Bari, 19-21 mai 1975), $2^{\mathrm{e}}$ éd. Bari, edizioni Dedalo, 1991 p. 223-280 ; I. Peri, Uomini, città e campagne in Sicilia dall'XI al XIII secolo, $2^{\mathrm{e}}$ éd. revue et corrigée, Rome/Bari, Laterza, 1990 p. 96-99 ; V. Von Falkenhausen, « Il popolamento : etnie, fedi, insediamenti ", dans G. Musca (éd.), Terra e uomini nel Mezzogiorno normanno-svevo. Atti delle settime giornate normanno-sveve (Bari, 15-17 octobre 1987), Bari, edizioni Dedalo, 1987, p. 39-73.

33. Sur Robert Avenel, E. Pontieri, «La madre... », p. 454-455 ; L.-R. Ménager, «Inventaire... », p. 378-379 ; Rogerii II diplomata...,éd. cit.,doc. II, 3, p. 6-8. Sur Josbert de Luci, C. A. Garufi, « Per la storia dei secoli XI e XII. Miscellanea diplomatica. III. La contea di Paternò e i de Luci ", dans Archivio storico per la provincia orientale, 10, 1913, p. 160-180, p. 172-173, note 4; L.-R. Ménager, «Inventaire... », p. 323-326. Sur Guillaume de Hauteville, Id., "L'abbaye bénédictine de la Trinité de Mileto en Calabre à l'époque normande ", dans Bullettino dell'archivio paleografico italiano., n. s., 4-5, 1958-1959, p. 9-94, p. 65, note 4 ; Id., «Inventaire... », p. 319 ; Rogerii II diplomata, éd. cit., doc. II, p. 5 .

34. Sur Nicolas, F. Giunta, Bizantini e bizantinismo nella Sicilia normanna, $2^{\mathrm{e}}$ éd. revue et corrigée, Palerme, Palumbo, 1974, p. 101-102. Sur Christodoule, L.-R. Ménager, «Amiratus : l'Amirat et les origines de l'Amirauté (XI ${ }^{\mathrm{e}-\mathrm{XII}}{ }^{\mathrm{e}}$ siècles) », Paris, SEVPEN, 1960, p. 28-36; V. Von Falkenhausen, « I 
ceti dirigenti prenormanni al tempo della costituzione degli stati normanni nell'Italia meridionale e in Sicilia ", dans G. Rossetti (éd.), Forme di potere e struttura sociale in Italia nel Medio Evo, Bologne, il Mulino, 1977, p. 321-377.

35. La résidence de Roger $\mathrm{I}^{\mathrm{er}}$ ne se trouvait pas à Mileto, mais à Troina, L.-R. Ménager, «L'abbaye... », p. 69.

36. Messine : doc. n. 7, n. 12, n. 13, n. 14, n. 20, n. 21 dans E. Caspar, Ruggero II e la fondazione della monarchia normanna di Sicilia, trad. de l'allemand par L. M. Iacono et M. Musetti, Centro europeo di Studi Normanni, Rome/Bari, Laterza, 1999, p.446-450; P. Collura, «Appendice al regesto dei diplomi di re Ruggero compilato da Erich Caspar », dans Atti stud ruggeriani..., vol. 2, p. 595-597, doc. n. 11, p. 559-560. San Marco d'Alunzio (province d'Enna): doc. n. 1 et n. 9, E. Caspar, Ruggero..., p. 445, p. 447. Troina : doc. n. 15, dans ibid., p. 448.

37. Le 12 juin 1112, la comtesse et son fils souscrivent un acte Panormi morantes... in thalamo superioris castri nostri : Rogerii II diplomata..., éd. cit., doc. II, 3, p. 6-8.

38. Les derniers documents souscrits à Messine par Adélaïde datent de janvier 1112 (P. Collura, "Appendice al regesto... », doc. n. 11, p. 559-561 ; doc. n. 2, p. 603-606) et de mars 1112 (I diplomi..., éd. cit., p. 407 ; E. Caspar, Ruggero..., doc. 20, p. 449).

39. Snorri Stulurson, Snorris Königsbuch. Heimskringla, trad. de l'islandais par F. Nieder, Iéna, E. Diederich, 1922 (Sammlung Thule II, 16), vol. 2, p. 216.

40. Guillaume de Tyr, Willermi Tyrensis archiepiscopi Historia rerum in partibus transmarinis gestarum, dansRecueil des Historiens des Croisades. Historiens occidentaux, éd. Académie des Inscriptions et des Belles Lettres, Paris, Imprimerie impériale, 1844/1897, vol. 5, XI, 21, p. 525.

41. Orderic Vital, Historia ecclesiastica, éd. cit., 6, XIII 15, p. 432.

42. Ibid., XI, 21, p. 526.

43. Ibid.., XI, 29, p. 54 : Interdixit etiam illi hac de causa quod consanguinitatis eiusdem matronae, ortae de sanguine Gallorum, reus haberetur.

44. Sur la mort d'Adélaïde, Annales siculi, éd. E. Pontieri, dans Geoffroi Malaterra, De rebus gestis..., éd. cit., p. 116 ; E. Winkelmann, « Reisefrüchte... », p. 472.

45. Sur ce sujet, R. La Duca, I veleni di Palermo, Palerme, Sellerio, 1988.

46. Petri Ansolini de Ebulo de rebus siculis carmen, éd. et notes d'E. Rota, Città di Castello, S. Lapi, 1904-1910, Rerum italicarum scriptores 31, 1-3 ; Liber ad honorem Augusti, éd., introd. et notes de G. B. Siragusa, Rome, Istituto storico italiano, 1906, Fonti per la storia d'Italia, 39, et Liber ad Honorem Augusti sive de rebus Siculis. Codex 120/II der Burgerbibliothek Bern. Eine Bilderchronik der Stauferzeit, éd. Th. Kölzer et M. Stähli, trad. G. Becht-Jördens, Sigmaringen, J. Thorbecke, 1994. Sur Pierre de Eboli, Studi su Pietro da Eboli, Rome, Istituto storico italiano per il Medio Evo, 1978.

47. G. B. Siragusa, «Le miniature che illustrano il carme di Pietro da Eboli nel cod. 120 della Biblioteca di Berna", Bollettino dell'Istituto storico italiano e Archivio muratoriano, 35, 1924, p. 115-163.

48. D. Jacquart et F. Micheau, La médecine arabe et l'Occident médiéval, Paris, Maisonneuve et Larose, 1996.

49. P. Kristeller, Studi sulla scuola medica salernitana, Naples, Istituto italiana per gli studi filosofici, 1986 ; M. Oldoni, «La Scuola medica di Salerno nella cultura europea fra IX e XIII secolo ", Quaderni medievali, 23, 1987, p. 74-93 ; M. Pasca (éd.), La scuola medica salernitana. Storia, immagini e manoscritti dall'XI al XIII secolo, Naples, Electa, 1987 et G. Vitolo, « Hippocratica civitas. Salerno e la scuola medica ", Tra Napoli e Salerno. La costruzione dell'identità cittadina nel Mezzogiorno medievale, Salerne, Carlone, 2001.

50. Sur ce sujet, Ch. H. Haskins, "Science at the Court of Emperor Frederick II ", Studies in the History of Medieval Science, New York, 1967; P. Toubert et A. Paravicini Bagliani, Federico II e le scienze, Palerme, Sellerio, 1995 et A. Varvaro, «Federico II e la cultura del suo tempo », Studi storici, 1, 1996, p. 391-404. 
51. M. S. Messana, Inquisitori, negormanti, streghe nella Sicilia moderna. 1500-1782, Palerme, Selerio, 2007.

52. V. La Mantia, Storia della legislazione criminale e civile di Sicilia, réimpr. anast., Bologne, A. Forni, 1991.

53. P. Sforza Pallavicino, Vita di Alessandro VII libri cinque del cardinale Sforza Pallavicino con discorso di Paolo Giordani sulla vita e sulle opere dell'autore,Milan,perG. Silvestri, 1853.

54. L. Natoli, La vecchia dell'aceto, Palerme, Flaccovio, 1991 et S. Salomone Marino, Leggende popolari siciliane in poesia, réimpr. anast., Bologne, A. Forni, 1998.

\section{RÉSUMÉS}

Cet article décrit quelques épisodes de la vie de la comtesse Adélaïde del Vasto, régente de comté de Calabre et de Sicile, telle qu'elle nous a été transmise par son principal biographe, Orderic Vital. La biographie du moine normand met en évidence l'un des thèmes dominants des écrits du célèbre chroniqueur: le crime d'empoisonnement perpétré par les femmes de pouvoir.

This article focuses on a few episodes in the life of Countess Adelaide del Vasto, regent of the county of Calabria and of Sicily, as recounted by her principal biographer Orderic Vitalis. In his chronicle, the Norman monk highlights the theme of poisoning by women in power.

\section{AUTEUR}

\section{BÉRANGÈRE SOUSTRE DE CONDAT-RABOURDIN}

Université catholique de Louvain. 